\title{
AFFILIATIVE TERMS AS A LINGUISTIC TOOL FOR IMPROVING PERCEPTIONS OF OUT-GROUP MEMBERS?
}

\author{
Cyril C. Grueter ${ }^{1,2^{*}}$, Ivan Roncevich ${ }^{{ }^{*}}$, Nicolas Fay ${ }^{3}$ \\ ${ }^{1}$ School of Human Sciences, The University of Western Australia, Perth, Australia \\ ${ }^{2}$ Centre for Evolutionary Biology, School of Biological Sciences, The University of Western \\ Australia, Perth, Australia \\ ${ }^{3}$ School of Psychological Science, The University of Western Australia, Perth, Australia
}

cyril.grueter@uwa.edu.au

\begin{abstract}
Group-contingent prosociality pervades human societies but the payoffs associated with intergroup tolerance and cooperation act as counterforces. Kinship terms (e.g. 'brother') and affiliative terms (e.g. 'mate') have been proposed to function to create strong bonds among in-group members but it is unknown if they play a role in establishing or strengthening between-group ties. To explore that possibility we conducted a psychological experiment whereby we recorded six speeches by three male speakers that differed along two lines: $i$ ) the accent of the speaker (Australian English, British English and English with a foreign accent), and ii) the inclusion of the word 'mate'. Listeners ( $N=90 ; 45$ males and 45 females) rated each vocal stimulus in terms of trustworthiness using a Likert scale. Our results support existing literature that subjects regard speakers with their own dialect as having higher trustworthiness. However, results do not accord with the notion that affiliative terms are harnessed to get access to social capital within and between groups. In sum, while accents can serve as reliable markers allowing selective assortment among individuals, affiliative terms do not seem to have such social utility (probably because they are easy to fake and thus do not constitute honest signals).
\end{abstract}

Keywords: Kinship terms, mateship, accent, out-group discrimination, social bonds

\footnotetext{
${ }^{*}$ These authors contributed equally to the paper
} 


\section{INTRODUCTION}

\section{"All people within the Four Seas [the world] are brothers" - Confucius}

In-group favouritism refers to a phenomenon whereby in-group members receive higher ratings in dimensions of trust, integrity, and honesty than out-group members and prosocial behaviours are more likely to be directed to in-group members than to out-group members (Brewer, 1979; Chen \& Li, 2009; Kramer, 1999; LeVine \& Campbell, 1972; Tajfel, Billig, Bundy, \& Flament, 1971; but see Grueter, Westlake, \& Coall, 2021; Saucier, Miller, \& Doucet, 2005). In-group favouritism is deeply rooted in human psychology, and while it can be considered a human universal, the expression of in-group preferences varies crossculturally (e.g. Hruschka \& Henrich, 2013; Romano, Balliet, Yamagishi, \& Liu, 2017). Ingroup preferences can be elicited regardless of whether group division occurs based on trivial criteria (such as preferences for particular painters (Yamagishi \& Mifune, 2008) or salient real world categories such as nationality, ethnicity, political affiliation or religion (Chuah, Fahoum, \& Hoffmann, 2013; Feather, 1994; Rand et al., 2009; Whitt \& Wilson, 2007).

One commonly invoked explanation for the evolution of in-group favouritism relies on the paradigm of cultural group selection: powered by the propagation of group-specific norms, a group of altruists is more likely to be competitive and victorious when confronted with between-group conflict (Boyd \& Richerson, 1990; Henrich, 2004; Richerson et al., 2016). Bounded generalized reciprocity constitutes another framework for explaining ingroup favouritism: here it is the prospect of establishing and maintaining a cooperative reputation and reciprocally altruistic relationships between repeatedly interacting individuals in distinct groups that drives preferential prosocial investments in in-group members (Kiyonari, Tanida, \& Yamagishi, 2000; Stroebe, Lodewijkx, \& Spears, 2005; Yamagishi, Jin, \& Kiyonari, 1999; but see Romano et al., 2017).

In large-scale societies with high levels of mobility and anonymity groups are demarcated by ethnic or cultural markers signalling membership (Moffett, 2013; Nettle \& Dunbar, 1997) and enabling selective assortment among individuals (McElreath, Boyd, \& Richerson, 2003; Riolo, Cohen, \& Axelrod, 2001). According to one line of thought, distinguishable group markers allow individuals to capitalize on the cooperative norms characterizing in-groups and, by directing cooperative behaviour selectively towards ingroup members, individuals can harness benefits from reciprocal exchanges (Nettle \& Dunbar, 1997). Experimental simulations demonstrate that group cooperative strategies between individuals who share a group marker are able to arise and proliferate under a wide range of conditions (Hammond \& Axelrod, 2006; Ihara, 2011; Riolo et al., 2001). Furthermore, results of laboratory one-shot economic games have shown that strangers who share group markers (even if arbitrarily assigned) are more likely to cooperate and act altruistically towards each other than participants who do not share a marker (Chen \& $\mathrm{Li}$, 2009; Hewstone, Rubin, \& Willis, 2002) [for a critical discussion of some of this empirical evidence, see Jensen, Petersen, Høgh-Olesen, \& Ejstrup, 2015]. An alternative account proposes that markers facilitate coordination among individuals in a heterogeneous population because they signal one's behavioural type/preferences/habits and allow unfamiliar individuals to identify others who share their social norms/cultural expectations (Gil-White, 2001; McElreath et al., 2003). Support for this conjecture comes from field research (Barth, 1969), laboratory studies (Efferson, Lalive, \& Fehr, 2008; Jensen et al., 2015) and modelling (McElreath et al., 2003).

Accent, i.e. one's manner of pronunciation (Giles, 1970), is a particularly pertinent and universal social marker (McElreath et al., 2003; Nettle \& Dunbar, 1997). Accent is a reliable cue of group membership as it is relatively invulnerable to imitation (and invasion by 
cheaters) and symbolises that group cleavage has occurred for a considerable period of time (Cohen et al., 2012; Richerson \& Boyd, 2001). Accent functions as an auditory index of social allegiance (Edwards, 2009; Lippi-Green, 1997) and is suitable for tag-based cooperation (Cohen et al., 2012). A rich body of sociolinguistic literature conducted across the globe has demonstrated how accent shapes group preferences; non-native/accented speakers are perceived less positively than native/local speakers in domains such as integrity and solidarity ((Edwards, 2009; Giles \& Powesland, 1975; Gluszek \& Dovidio, 2010; Lindemann, 2003; Trudgill, 1974; but see Jensen et al., 2015). This is either because accented speakers produce prejudicial reactions or because they are harder to understand (Lev-Ari \& Keysar, 2010). Accents not only influence cognitive ratings but also economic decisions such as whether to cooperate or compete (Heblich, Lameli, \& Riener, 2015). Accents are also instrumental in directing infants' and childrens' social preferences, more so than other social categories such as race (Kinzler, Shutts, DeJesus, \& Spelke, 2009). The origin of accents is unclear. They may have already played a role as social information signals in dealings with neighboring societies in the Middle Stone Age, in a similar vein to what has been proposed for cultural artifacts (Kuhn \& Stiner 2007).

While in-group favouristism and parochial altruism are parts of humans' evolutionary legacy, tolerant and cooperative relationships between groups are trademarks as well, and may be mutually advantageous in the context of trade, military alliances etc. (Grueter \& White, 2014; Pisor \& Surbeck, 2019; Schaub, 2017). These large-scale cooperative endeavours are predominantly the domain of males who differ in their cooperative profile from females, with females concentrating their cooperative activities on a smaller set of same-sex individuals (Maccoby 1998; Tiger 2005; Wrangham \& Benenson 2017). Given the salience of accent as a structuring principle of social preferences, it is unclear how sociolinguistic barriers can proximately be overcome and between-group coordination realized. We speculate that fictive kinship (the use of kin terms to label others who are genetically unrelated, such as 'brother') or affiliative terms used to address someone (e.g. 'comrade' or 'mate') may have been co-opted for transcending group boundaries. There is little hypothesis-driven empirical work on the evolutionary significance of such terms but they seem to strengthen ties within distinct social groups. It has been hypothesized that the metaphorical use of kinship terms can "exploit the natural solidarity of kin or to tap into the emotions/bonds/obligations of kinship" (Salmon, 1998, p. 52). Kin terms contribute to commitment to particular communities (religious or secular, including militant groups), facilitate social coordination and are used to reinforce cooperative norms of parochial altruism and patriotism (Abou-Abdallah, Kashima, \& Harb, 2016; Bogin, Bragg, \& Kuzawa, 2014; Carsten, 2000; Johnson, 1987; McNamara \& Henrich, 2016; Norenzayan et al., 2016; Qirko, 2013; Salmon, 1998; see also Cronk, Steklis, Steklis, van den Akker, \& Aktipis, 2019; Daly, Salmon, \& Wilson, 1997). Whether kinship or affiliative terminology can be used to overcome out-group stigmatization has to our knowledge not been addressed. Given the prevalence of inter-group conflict and discrimination based on group identity this research is timely and of significant applied value as it explores ways of attenuating parochialism.

One affiliative term commonly used in Australia is 'mate.' 'Mateship', which derives from 'mate', refers to an Australian code of conduct that accentuates egalitarianism, loyalty and friendship. Historically the concept of mateship can be traced to the early convict settlers as well as Australian soldiers serving in WWI. Particularly during early colonial times the harsh environmental conditions encountered by settlers instilled into them a sense of we-ness and made mutual assistance mandatory. Over time, different connotations have been attached to mateship, including socialism, patriotism, fellowships, and business partnerships (Dyrenfurth, 2015). The term 'mate' is frequently used to address friends, colleagues and strangers. 
The first aim of the current study is to examine the effect of accent on pro-social assessment. We predict that individuals will perceive others with similar accents as more trustworthy than those with different accents. The second aim is to determine whether the figurative use of affiliative terms modulates perceptions of both in-group and out-group speakers, and to what extent. We predict that perception of a speaker's trustworthiness is enhanced through this terminological practice. Lastly, we aim to determine whether males are more responsive to affiliative terms and more susceptible to altering their perceptions of out-group individuals than females.

\section{METHODS}

\section{Data collection}

Participants

A convenience sample of 90 university students took part in the study on a voluntary basis. All students were second generation Australian born citizens, and were enrolled in various majors at the University of Western Australia. Of these, 45 were male and 45 were female. All participants were between 18 and 27 years of age (with an average of 21) and of Caucasian descent. The recruitment procedure for participants in this experiment was designed to ensure that only individuals with standard Australian accents would take part in the study without the participants themselves being aware of this fact. Furthermore, to increase ecological validity, recruitment was designed to ensure that participants did not consider themselves participants of a laboratory experiment, but instead participants of a recruitment panel.

\section{Experimental design}

The study took place in July and August 2019 at the University of Western Australia. Participants were verbally briefed on the nature of the experiment. Temporary deception was used to ensure a realistic experimental design. Participants were falsely led to believe that the experiment was being conducted on behalf of an anonymous outdoor adventuring company and that they were assessing the trustworthiness of potential job applicants for an extreme sports (bungee jumping) instructor role. The experiment consisted of listening to six pre-recorded speeches of 'applicants' and assessing the trustworthiness of the speakers on a 7-point Likert scale. We used only male speakers because of the putative evolutionary and historical importance of affiliative terms in the context male-male bonding/ cooperation. The experiment took approximately 15 minutes in total. After completing the experiment subjects were debriefed on the real aims of the experiment and the use of deception.

\section{Vocal stimuli}

Three differently accented male speakers (all in their 20s) were used to create vocal stimuli. Speaker accents were standard Australian English, a native English accent (British English), and English with a foreign accent (Swedish). Each speaker was given two versions of a template speech to recite: a version infused with the word 'mate' and one without (see Supplementary text S1). Aside from inclusion of the word 'mate' at the beginning and end, each speech was identical. We used a Hi-Q MP3 Voice Recorder installed on an Android 
device and an external microphone. Each speech was approximately 45 seconds in length. We ensured that speaking speed, loudness, tone and emphasis remained consistent across stimuli. The three speakers featured a similar vocal pitch (between $140-160 \mathrm{~Hz}$ ) which guaranteed control of these potentially confounding individual characteristics. All six vocal stimuli were played to each participant. Vocal stimuli of the same speaker were never presented in succession. Vocal stimuli were delivered to participants in a semi-random order to account for any unwanted bias which may have developed over the course of the experiment.

\section{Data analysis}

All data obtained can be found in the supplementary table S2. R version 3.6.0 was used for all the analyses (R Core Team, 2019). We constructed liner mixed effects model with a Gaussian error structure and identity link function in the package lme4 (Bates, Maechler, Bolker, \& Walker, 2015). Trustworthiness ratings were modelled as the dependent variable. Fixed effects were accent (factor with three levels), and affiliative terms (dichotomous variable). Listener sex (dichotomous variable) and stimulus order (i.e., the order in which a sequence of stimuli was presented; factor with four levels) were entered as control predictors. Random intercepts were specified for each listener to control for repeated measures. The model did not converge with random slopes, so we ran it without them. To examine potential collinearity among the explanatory terms, we computed variance inflation factors (VIF) using a standard linear model without the random effects (using the function 'vif' of the R package car (Fox, 2011). All VIFs were $<2$ of which is well below recommended cut-offs (Quinn \& Keough, 2002; Zuur, Ieno, \& Elphick, 2010).

We used an information-theoretic model selection approach based on the Akaike information criterion corrected for small sample sizes (AICc; Burnham \& Anderson, 2002). After specifying the saturated model that included all predictors we used the 'dredge' function in the package MuMIn (Barton, 2016) to generate and rank a set of all possible additive candidate models with the above predictors. Following the suggestion of Harrison et al. (2018) and Richards (2005), a shortlist of competitive models with $\Delta_{i}<6$ was created. Next, to account for uncertainty inherent in model selection, we performed model averaging on the above 'confidence set' (Burnham \& Anderson, 2002; Nettle, 2019; Symonds \& Moussalli, 2011) using the function 'model.avg' in MuMIn. Averaged parameter estimates and associated standard errors and 95\% confidence intervals were derived from the Akaike weights $\left(\mathrm{w}_{i}\right)$ of each model.

For each of the shortlisted models, we also calculated marginal (variance explained by the fixed effects) and conditional (variance explained by the entire model, including both fixed and random effects) coefficients of determination following Nakagawa \& Schielzeth (2013) and Johnson (2014). $\mathrm{R}^{2}$ was calculated using the function 'r.squaredGLMM' in the R package MuMIn.

\section{Ethics}

The study received approval from the University of Western Australia Ethics Committee. Participants viewed an information sheet before giving written consent to take part in the study. The information sheet and consent form were both approved by the Ethics Committee. All methods were performed in accordance with the guidelines from the National Health and Medical Research Council/Australian Research Council/University Australia's National Statement on Ethical Conduct in Human Research. 


\section{RESULTS}

The best-models subset (based on the $\Delta_{i}<6$ cut-off criterion) contained a model with accent as the sole predictor (AICc weight $=0.784$ ), a model with accent and sex as predictors (AICc weight $=0.128$ ) and a model with accent and affiliative terms as predictors $($ AICc weight $=0.059)($ Table 1$)$. The variable 'stimulus order' did not appear in any of the highly ranked models. Model averaging based on a set of plausible models show that the Australian English speaker received higher trustworthiness ratings than the British English speaker and foreign-accented English speaker (Table 2). The trustworthiness ratings of the British English speaker were virtually indistinguishable from those of the Swedish English speaker (Fig. 1a). The results do not support an association between the use of affiliative terms and perceptions of trustworthiness (Fig. 1b).

Table 1: Results of a GLMM examining variables associated with trustworthiness.

\begin{tabular}{lccccccc}
\hline Model & $\mathrm{df}$ & $\operatorname{logLik}$ & $\mathrm{AICc}$ & $\Delta_{i}$ & $\mathrm{w}_{i}$ & $\mathrm{R}^{2}$ marginal & $\mathrm{R}^{2}$ conditional \\
\hline Accent & 5 & -792.327 & 1594.8 & 0 & 784 & 0.084408214 & 0.333425 \\
Accent + Sex & 6 & -793.116 & 1598.4 & 3.62 & 128 & 0.08563099 & 0.336084 \\
Accent + affiliative terms & 6 & -793.883 & 1599.9 & 5.16 & 59 & 0.08430153 & 0.332803 \\
\hline
\end{tabular}

$\mathrm{df}=$ degrees of freedom; $\log \mathrm{Lik}=\log$ likelihoods; AICc = Akaike's Information criterion corrected for small sample sizes; $\mathrm{w}_{i}=$ Akaike weights; $\Delta_{i}=$ difference in AICc value between top model and $i$ th model.

Table 1: Weighted mean parameter estimates derived from the candidate model set in Table

\begin{tabular}{llllll}
\hline Parameter & Estimate & Adjusted SE & $\mathrm{z}$ & $\operatorname{Pr}(>|\mathrm{z}|)$ & $95 \%$ CIs \\
\hline (Intercept) & 5.648 & 100 & 56.206 & & $5.451,5.844$ \\
Accent (Swedish) & -810 & 102 & 7.979 & $<0.0001$ & $-1.009,-0.611$ \\
Accent (UK) & -575 & 102 & 5.669 & $<0.0001$ & $-0.774,-0.376$ \\
Sex (male) & -12 & 63 & 197 & 844 & $-0.386,0.199$ \\
Affiliative term (no) & 0 & -21 & 11 & 991 & $-0.162,0.170$ \\
\hline
\end{tabular}

$\mathrm{SE}=$ standard error; $\mathrm{CI}=$ confidence intervals. 

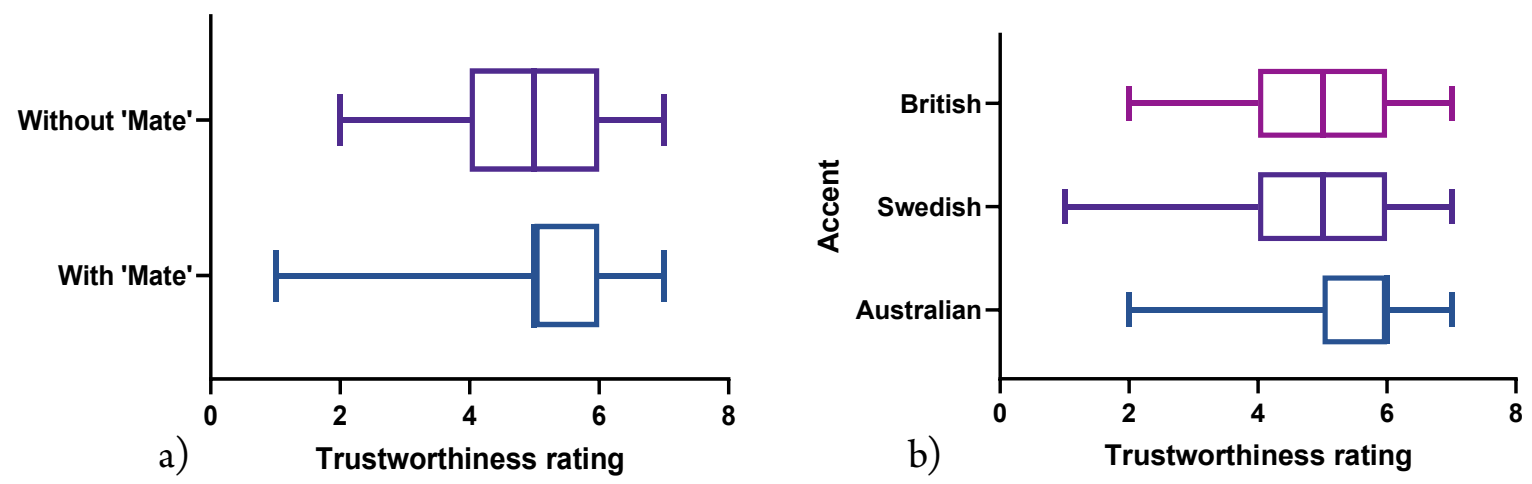

Fig. 1: a) Association between use vs. non-use of affiliative terms and trustworthiness ratings. b) Association between accent and trustworthiness ratings. Vertical lines denote median, $1^{\text {st }}$ and $3^{\text {rd }}$ quartiles.

\section{DISCUSSION}

The results of our study show that the statistical model with accent alone had the highest predictive accuracy. This supports the prediction that subjects regard speakers with their own accent as having higher trustworthiness and that phonetic deviation from local accent can cause less favourable perceptions. There is ample evidence from English-speaking countries that people who speak with a non-native accent are perceived more negatively than speakers with native accents (Brennan and Brennan 1981; Callan et al. 1983; Fuertes et al. 2012; Giles 1970). Our findings underscore that accent is a fundamental and salient social identity cue and a reliable marker of group affiliation in large dynamic social settings (Cohen et al. 2012; Gluszek and Dovidio 2010). Despite geographical mobility and intermixing on a global scale, accent still affects listeners' judgments of speakers' trustworthiness. By inference, one would expect social categorization based on linguistic tags to have been even more pronounced before globalization and long-distance travel.

An alternative explanation for the more negative perception and assessment of speakers of an accent other than Australian-English is that it may be more difficult to process and understand the verbal utterings of a speaker of an unfamiliar accent (Lev-Ari \& Keysar, 2010). However, the likely collinearity between accent and comprehension may make statistically differentiating between these two explanatory variables tricky. Moreover, in the context of our study, the fact that the British English speaker was indistinguishable in terms of trustworthiness ratings from the Swedish English speaker speaks against a confounding effect of comprehension.

Metaphorically invoking affiliation via the affiliative term 'mate' did not induce changes in perceptions of a speaker's trustworthiness. Neither males nor females assigned higher levels of trustworthiness to speakers using 'mate', irrespective of accent. This finding does not support the prediction that affiliative terminology is harnessed to get access to social capital within and between groups. One possible reason that affiliative terms did not elicit higher trustworthiness ratings for dyads sharing the same dialect is that affiliative terms were historically adaptive but have become vestigial in modern environments. However, the scarce evidence on kin terms contradicts this and instead suggests socio-environmental and political context may modulate the importance of kin terms in structuring social perceptions and relationships. Salmon (1998) found that kin terms were effective in evoking listeners' 
support for the political views expressed in the speech that the subjects heard. The absence of an effect of affiliative terms in our study may be because our study was not couched in a political context. Moreover, Abou-Abdallah et al. (2016) report that approval of parochially altruistic violence by an in-group against an out-group was associated with the use of kinship metaphors among in-group members such as 'brothers' but this effect was only apparent when the out-group posed a threat to the in-group; that outgroup threat element was missing from our study design and may well have been the tipping point for eliciting a change in evaluation. It is also possible that the failure to detect any social utility of affiliative terms in this study is because affiliative terms are - contrary to accents - easy to fake and thus do not constitute honest signals. Yet another possibility for the listeners' unresponsiveness to the usage of the term 'mate' is that it is so universally used in Australia these days that it may have lost its efficacy.

Moreover, the importance of affiliative terms in inducing a sense of belonging may depend on how stressful a particular environment is. Although this is purely anecdotal, the use of the word 'mate' in Australia seems to be linked to socio-economic status and educational background. Conducting similar studies across a socioeconomic gradient would shed light on such possible ecological variation. Furthermore, given that social class can affect trustworthiness (Korndörfer, Egloff, \& Schmukle, 2015), it would be prudent to incorporate the listeners' socioeconomic background as an explanatory variable in future iterations of this experiment. Another variable to consider when refining our methodological approach is the demographics of the population under study, as affiliative terms may be more important in certain age categories. Approaching the question of the role of affiliative and kinship terms in facilitating social cohesion from a cross-cultural/ cross-societal perspective would also be a valuable direction for future research considering that the WEIRD (Western, educated, industrialized, rich, democratic) population used in the present study may not be representative of humanity as a whole (Gurven, 2018).

Our study design is derived from the implicit assumption that 'mateship' is functionally similar to kinship psychology even though the term does not describe a genealogical relationship. To validate the use of 'mate' as a surrogate kin term a replication of the study with other kin terms (e.g. 'bro' which is commonly used among African-Americans and also other groups such as American fraternity men, surfers and gang members) would be needed. In this study the only outcome variable we measured was trustworthiness. Followup studies may examine how kinship terms influence other variables such as coordination and cooperation. Moreover, the empirical question of the functional significance of kin terms can also be explored with economic games such as the trust game (Berg, Dickhaut, \& McCabe, 1995).

Our research also allows us to hone in on question of what constitutes a sociolinguistic group and which English accents convey a common connection (Gluszek \& Dovidio, 2010): are all native English speakers (irrespective of whether they are British, Australian etc.) members of the same group, or do we draw the boundary at the level of the variety of the native accent? Our results indicate that Australian English speakers represent the ingroup whereas other English speakers are the out-group. In other words, there is no superordinate entity of native English accents.

The finding that linguistic group delineation moulds perceptions of trustworthiness makes it plausible that trust drives in-group cooperation. However, this would defy the argument that individuals channel cooperative and prosocial efforts to in-group members to enhance their personal reputation within the group (Yamagishi \& Mifune, 2008; see also (Jensen et al., 2015).

Our experimental design did not draw on the matched guise technique according to which the three language variety samples would have had to be produced by the same 
speaker (Lambert, 1967). The matched guise technique controls for speaker-specific features (e.g. pitch) that could muddle the results if different speakers were used. For example, vocally expressed confidence through pitch and loudness contains information about a speaker's trustworthiness (Jiang, Sanford, \& Pell, 2018). It would have been virtually impossible to find a speaker who can convincingly and faithfully produce all three accents (e.g. Clarke \& Garrett, 2004). We therefore asked participants to evaluate accents spoken by different speakers and thereby risked the possibility that listeners were reacting to traits other than accent. However, we tried to limit the effect of this possible confound by a priori selecting speakers with limited variation in vocal expression.

In conclusion, we found that accent influenced perceptions of trustworthiness among in- and out-group members in our study, but that adding the affiliative term 'mate' did not. However, replication across a variety of contexts is needed to establish whether affiliative terms can potentially be effective in surmounting out-group prejudice. Our finding that accented speakers receive lower trustworthiness ratings from local speakers emphasizes the need to take the prevention of accent-based discrimination seriously.

\section{SUPPLEMENTARY MATERIAL}

Electronic supplementary materials are available online.

\section{REFERENCES}

Abou-Abdallah, M., Kashima, Y., \& Harb, C. (2016). "Brothers" in Arms: Does metaphorizing kinship increase approval of parochial altruism? Journal of Cognition and Culture, 16, 37-49. DOI

Barth, F. e. (1969). Ethnic groups and boundaries: The social organization of culture difference. Boston: Little, Brown.

Barton, K. (2016). MuMIn: Multi-model inference. R package version 1.15. 6. Available from: https://cran.r-project.org/web/packages/MuMIn/index.html.

Bates, D., Maechler, M., Bolker, B., \& Walker, S. (2015). Fitting linear mixed-effects models using lme4. Journal of Statistical Software, 67, 1-48.

Berg, J., Dickhaut, J., \& McCabe, K. (1995). Trust, reciprocity, and social history. Games and Economic behavior, 10(1), 122-142. DOI

Bogin, B., Bragg, J., \& Kuzawa, C. (2014). Humans are not cooperative breeders but practice biocultural reproduction. Annals of Human Biology, 41(4), 368-380. DOI

Boyd, R., \& Richerson, P. J. (1990). Group selection among alternative evolutionarily stable strategies. Journal of Theoretical Biology, 145(3), 331-342. DOI

Brewer, M. B. (1979). Ingroup bias in the minimal intergroup situation: A cognitivemotivational analysis. Psychological Bulletin, 86(2), 307-324. DOI

Burnham, K. P., \& Anderson, D. R. (2002). Model selection and multimodel inference: A practical information-theoretic approach. New York: Springer. DOI

Carsten, J. (Ed.) (2000). Cultures of relatedness: New approaches to the study of kinship. Cambridge: Cambridge University Press.

Chen, Y., \& Li, S. X. (2009). Group identity and social preferences. American Economic Review, 99(1), 431-457. DOI

Chuah, S. H., Fahoum, R., \& Hoffmann, R. (2013). Fractionalization and trust in India: A field-experiment. Economics Letters, 119(2), 191-194. DOI 
Clarke, C. M., \& Garrett, M. F. (2004). Rapid adaptation to foreign-accented English. The Journal of the Acoustical Society of America, 116(6), 3647-3658. DOI

Cohen, E., Atkinson, Q. D., Dediu, D., Dingemanse, M., D. Kinzler, K., Ladd, D. R., . . . Trudgill, P. (2012). The evolution of tag-based cooperation in humans: The case for accent. Current Anthropology, 53(5), 588-616. DOI

Cronk, L., Steklis, D., Steklis, N., van den Akker, O. R., \& Aktipis, A. (2019). Kin terms and fitness interdependence. Evolution and Human Behavior, 40(3), 281-291. DOI

Daly, M., Salmon, C., \& Wilson, M. (1997). Kinship: The conceptual hole in psychological studies of social cognition and close relationships. In J. A. Simpson \& D. T. Kenrick (Eds.), Evolutionary Social Psychology (pp. 265-296). Mahwah, NJ: Erlbaum.

Dyrenfurth, N. (2015). Mateship: A very Australian history. Melbourne: Scribe.

Edwards, J. (2009). Language and identity. Cambridge: Cambridge University Press.

Efferson, C., Lalive, R., \& Fehr, E. (2008). The coevolution of cultural groups and ingroup favoritism. Science, 321(5897), 1844-1849. DOI

Feather, N. T. (1994). Values, national identification and favouritism towards the in-group. British Journal of Social Psychology, 33(4), 467-476. DOI

Fox J, W. S. (2011). An R companion to applied regression. Thousand Oaks: Sage.

Gil-White, F. J. (2001). Are ethnic groups biological "species" to the human brain? Essentialism in our cognition of some social categories. Current Anthropology, 42(4), 515-554. DOI

Giles, H. (1970). Evaluative reactions to accents. Educational Review, 22(3), 211-227. DOI

Giles, H., \& Powesland, P. F. (1975). Speech style and social evaluation. London: Academic.

Gluszek, A., \& Dovidio, J. F. (2010). The way they speak: a social psychological perspective on the stigma of nonnative accents in communication. Personality and Social Psychology Review, 14(2), 214-237. DOI

Grueter, C. C., Westlake, G., \& Coall, D. (2021). Absence of intergroup discrimination in a naturalistic helping task. Evolutionary Behavioral Sciences, 15(1), 82-88. DOI

Grueter, C. C., \& White, D. R. (2014). On the emergence of large-scale human social integration and its antecedents in primates. Structure and Dynamics, $7(1), 1-27$. DOI

Gurven, M. D. (2018). Broadening horizons: Sample diversity and socioecological theory are essential to the future of psychological science. Proceedings of the National Academy of Sciences, 115(45), 11420-11427. DOI

Hammond, R. A., \& Axelrod, R. (2006). The evolution of ethnocentrism. Journal of Conflict Resolution, 50, 926-936. DOI

Harrison, X. A., Donaldson, L., Correa-Cano, M. E., Evans, J., Fisher, D. N., Goodwin, C. E., ... Inger, R. (2018). A brief introduction to mixed effects modelling and multimodel inference in ecology. PeerJ, 6, e4794. DOI

Heblich, S., Lameli, A., \& Riener, G. (2015). The effect of perceived regional accents on individual economic behavior: A lab experiment on linguistic performance, cognitive ratings and economic decisions. PLoS ONE, 10(2), e0113475. DOI

Henrich, J. (2004). Cultural group selection, coevolutionary processes and large-scale cooperation. Journal of Economic Behavior and Organization, 53(1), 3-35. DOI

Hewstone, M., Rubin, M., \& Willis, H. (2002). Intergroup bias. Annual Review of Psychology, 53(1), 575-604. DOI

Hruschka, D. J., \& Henrich, J. (2013). Institutions, parasites and the persistence of in-group preferences. PLoS ONE, 8, e63642. DOI

Ihara, Y. (2011). Evolution of culture-dependent discriminate sociality: A gene-culture coevolutionary model. Philosophical Transactions of the Royal Society B: Biological Sciences, 266(1566), 889-900. DOI 
Jensen, N. H., Petersen, M. B., Høgh-Olesen, H., \& Ejstrup, M. (2015). Testing theories about ethnic markers: Ingroup accent facilitates coordination, not cooperation. Human Nature, 26, 210-234. DOI

Jiang, X., Sanford, R., \& Pell, M. D. (2018). Neural architecture underlying person perception from in-group and out-group voices. NeuroImage, 181, 582-597.

Johnson, G. R. (1987). In the name of the Fatherland: An analysis of kin term usage in patriotic speech and literature. International Political Science Review, 8, 165-174. DOI

Johnson, P. C. (2014). Extension of Nakagawa \& Schielzeth's R2 ${ }_{\text {GLMM }}$ to random slopes models. Methods in Ecology and Evolution, 5(9), 944-946. DOI

Kinzler, K. D., Shutts, K., DeJesus, J., \& Spelke, E. S. (2009). Accent trumps race in guiding children's social preferences. Social Cognition, 27(4), 623-634. DOI

Kiyonari, T., Tanida, S., \& Yamagishi, T. (2000). Social exchange and reciprocity: confusion or a heuristic? Evolution and Human Behavior, 21(6), 411-427. DOI

Korndörfer, M., Egloff, B., \& Schmukle, S. C. (2015). A large scale test of the effect of social class on prosocial behavior. PLoS ONE, 10(7): e0133193. DOI

Kramer, R. M. (1999). Trust and distrust in organizations: Emerging perspectives, enduring questions. Annual Review of Psychology, 50(1), 569-598. DOI

Kuhn, S. L., \& Stiner, M. (2007). Paleolithic ornaments: implications for cognition, demography and identity. Diogenes, 54(2), 40-48. DOI

Lambert, W. E. (1967). A social psychology of bilingualism. Journal of Social Issues, 23(2), 91-109. DOI

Lev-Ari, S., \& Keysar, B. (2010). Why don't we believe non-native speakers? The influence of accent on credibility. Journal of Experimental Social Psychology, 46(6), 1093-1096. $\underline{\mathrm{DOI}}$

LeVine, R. A., \& Campbell, D. T. (1972). Ethnocentrism: Theories of conflict, ethnic attitudes, and group behavior. New York: Wiley.

Lindemann, S. (2003). Koreans, Chinese or Indians? Attitudes and ideologies about nonnative English speakers in the United States. Journal of Sociolinguistics, 7(3), 348-364. DOI

Lippi-Green, R. (1997). English with Accents: Language, Ideology, and Discrimination in the United States. New York: Routledge.

Maccoby, E. E. (1998). The two sexes: Growing up apart, coming together. Cambridge, MA: Belknap Press/Harvard University Press.

McElreath, R., Boyd, R., \& Richerson, P. J. (2003). Shared norms and the evolution of ethnic markers. Current Anthropology, 44(1), 122-129. DOI

McNamara, R. A., \& Henrich, J. (2016). Kin and kinship psychology both influence cooperative coordination in Yasawa, Fiji. Evolution and Human Behavior, 38(3), 197-207. DOI

Moffett, M. W. (2013). Human identity and the evolution of societies. Human Nature, 24, 219-267. DOI

Nakagawa, S., \& Schielzeth, H. (2013). A general and simple method for obtaining R2 from generalized linear mixed-effects models. Methods in Ecology and Evolution, 4(2), 133-142. DOI

Nettle, D. (2019). Modelling and visualizing data using R: A practical introduction. https:// www.danielnettle.org.uk/r-modelling/

Nettle, D., \& Dunbar, R. I. M. (1997). Social markers and the evolution of reciprocal exchange. Current Anthropology, 38(1), 93-99. DOI

Norenzayan, A., Shariff, A. F., Gervais, W. M., Willard, A. K., McNamara, R. A., Slingerland, E., \& Henrich, J. (2016). The cultural evolution of prosocial religions. Behavioral and Brain Sciences, 39, e1. DOI 
Pisor, A. C., \& Surbeck, M. (2019). The evolution of intergroup tolerance in nonhuman primates and humans. Evolutionary Anhropology, 28(4), 210-223. DOI

Qirko, H. N. (2013). Induced altruism in religious, military, and terrorist organizations. Cross-Cultural Research, 47(2), 131-161. DOI

Quinn, G. P., \& Keough, M. J. (2002). Experimental Design and Data Analysis for Biologists. Cambridge: Cambridge University Press. DOI

R Core Team (2019). R: A Language and Environment for Statistical Computing. Vienna, Austria: R Foundation for Statistical Computing.

Rand, D. G., Pfeiffer, T., Dreber, A., Sheketoff, R. W., Wernerfelt, N. C., \& Benkler, Y. (2009). Dynamic remodeling of in-group bias during the 2008 presidential election. Proceedings of the National Academy of Sciences, 106(15), 6187-6191. DOI

Richards, S. A. (2005). Testing ecological theory using the information-theoretic approach: examples and cautionary results. Ecology, 86(10), 2805-2814. DOI

Richerson, P. J., Baldini, R., Bell, A. V., Demps, K., Frost, K., Hillis, V., . . . Zefferman, M. (2016). Cultural group selection plays an essential role in explaining human cooperation: A sketch of the evidence. Behavioral and Brain Sciences, 39, e30. DOI

Richerson, P. J., \& Boyd, R. (2001). The evolution of subjective commitment to groups: A tribal instincts hypothesis. In R. M. Nesse (Ed.), Evolution and the Capacity for Commitment (pp. 186-220). New York, NY: Russel Sage Foundation.

Riolo, R. L., Cohen, M. D., \& Axelrod, R. (2001). Evolution of cooperation without reciprocity. Nature, 414, 441-443. DOI

Romano, A., Balliet, D., Yamagishi, T., \& Liu, J. H. (2017). Parochial trust and cooperation across 17 societies. Proceedings of the National Academy of Sciences, 114(48), 1270212707. DOI

Salmon, C. (1998). The evocative nature of kin terminology in political rhetoric. Politics and the Life Sciences, 17, 51-57. DOI

Saucier, D. A., Miller, C. T., \& Doucet, N. (2005). Differences in helping whites and blacks: A meta-analysis. Personality and Social Psychology Review, 9(1), 2-16. DOI

Schaub, M. (2017). Threat and parochialism in intergroup relations: Lab-in-the-field evidence from rural Georgia. Proceedings of the Royal Society B, 284, 20171560. DOI

Stroebe, K., Lodewijkx, H. F. M., \& Spears, R. (2005). Do unto others as they do unto you: Reciprocity and social identification as determinants of ingroup favoritism. Personality and Social Psychology Bulletin, 31(6), 831-845. DOI

Symonds, M. R., \& Moussalli, A. (2011). A brief guide to model selection, multimodel inference and model averaging in behavioural ecology using Akaike's information criterion. Behavioral Ecology and Sociobiology, 65(1), 13-21. DOI

Tajfel, H., Billig, M. G., Bundy, R. P., \& Flament, C. (1971). Social categorization and intergroup behaviour. European Journal of Social Psychology, 1(2), 149-178. DOI

Tiger, L. (2005). Men in groups. Piscataway, NJ: Transaction.

Trudgill, P. (1974). Sociolinguistics. Harmondsworth: Penguin.

Whitt, S., \& Wilson, R. K. (2007). The dictator game, fairness and ethnicity in postwar Bosnia. American Journal of Political Science, 51(3), 655-668. DOI

Wrangham, R. W. \& Benenson, J. (2017). Cooperative and competitive relationships within sexes. In M. N. Muller, R. W. Wrangham \& D. R. Pilbeam (Eds.), Chimpanzees and human evolution (pp. 509-547). Cambridge, MA: Belknap Press/Harvard University Press. DOI

Yamagishi, T., Jin, N., \& Kiyonari, T. (1999). Bounded generalized reciprocity: Ingroup boasting and ingroup favoritism. Advances in Group Processes, 16(1), 161-197.

Yamagishi, T., \& Mifune, N. (2008). Does shared group membership promote altruism? Fear, greed, and reputation. Rationality Society, 20(1), 5-30. DOI 
Zuur, A. F., Ieno, E. N., \& Elphick, C. S. (2010). A protocol for data exploration to avoid common statistical problems. Methods in Ecology and Evolution, 1(1), 3-14. DOI 